CIHM

Microfiche Series (Monographs)
ICMH

Collection de microfiches (monographies)

Canadian Inatitute for Mistorical Microreproductions / Institut canadien de microreproductiona historiques
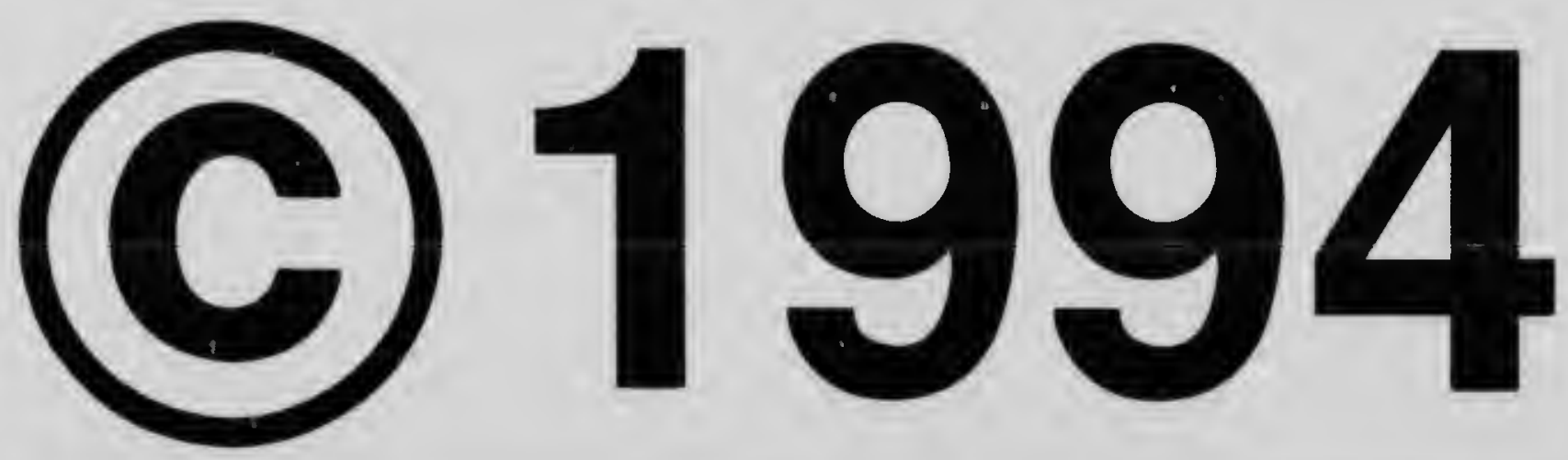
Technical and Bibliographic Notes / Notes techniques et bibliographiques

The Institute has attompted to obtain the best original copy available for filming. Features of this copy which may be bibliographically unique, which may atear any of the imeres in the reproduction, or which may significantly change the usual method of filming, are cheeked below.

Coloured covers/

Couverture de couleur

Covers damaend

Couverture endommegie

Covers restored and/or Iaminatad/

Couvurture restaurite et/ou polliculde

Cover title missing/

Le titre de couverture menque

Coloured meps/

Cartes gisographiques en couleur

Coloured ink (i.e. other then blue or black)/

Encre de couleur (i.e. autre que blewe ou noire)

Coloured plates and/or illustrations/

Planches et/ou illustrations en couleur

Bound with ether material/

Relib avec d'aytres documents

Tight binding may cause shedows or distortion slong interior margin/

Lo reliure serré paut causer de l'ombre ou de la distorsion le long de la marge intírieure

Blank leaves edded during restoration may appanr within the text. Whenever possible, these have been omitted from filming/

II sa peut que certaines paes blanches ajoutles lors d'ume restauration apparaissent dans le texte, mais, lorsque cela était possible. cas pags niont pas exé filmies.
L'Institut a microfilmó le meilleur exemplaire qu'il lui a bre possible de se procurer. Les ditails de cet exemplaire qui sont peut-tre uniques du point de vue bibliographique, qui peuvent modifier um imen reproduite, ou qui peuvent exiger une modifieation dons la mithode nomale de filmees somt indiquís ci-dessous.

Coloured peges/

Pages de couleur

Pages domaend/

Paces endomracies

Pages restored and/or laminated/

Pages restauries et/ou polliculies

7 Pages discoloured. stained or foxed/

Pages dieolories, tacheties ou piquies

Pages detached/

Pages dítachies

Showthrough/

Tramsparence

Quality of print varies/

Qualité incigale de l'impression

Continuous pagination/

Pagination continue

Includes index(es)/

Comprend un (des) index

Titte on heoder taken from:/

Le titre de l'en-the proviens:

Title page of issue/

Page de titre de la livraison

Caption of issuel

Tizre de depart de la livraison

Masthead/

Générique (périodiques) de la livraison

Additional comments:/

Commentaires supplémentaires:

This item is filmed at the reduction ratio checked below/ Ce document est filmé au taux de riduction indique ci-dessous.

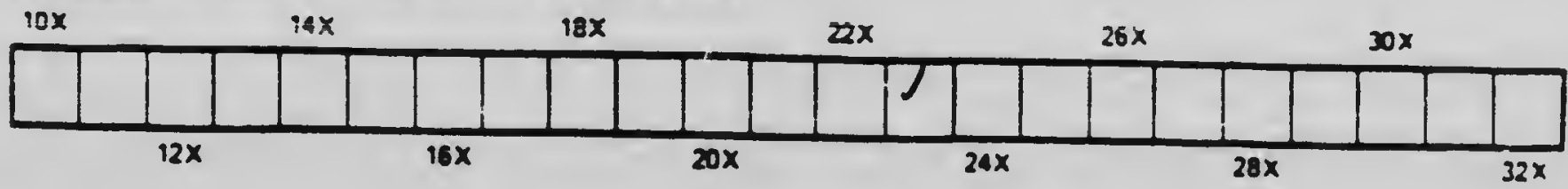


The copy filmed here hes been reproduced thank: to the ganeroaity of:

\section{Library}

Agriculture Canada

The Imagee appeering here are the bext queitty posalbie considering the condition and legibility of the originai copy and in keeping with the filming contraet epecifications.

Originai copies in printed paper covere are filmed beginning with the fromt cover and ending on the last page with a printed of illustrated impres. sion. of the back cover when eppropriate. All other original coples are filmed beginning on the first page with a printed or lilustrated impree. sion, and ending on the last page with a printed or iliustrated Impreacion.

The last recorded freme on eseh microfiche shall contuin the symbol $\rightarrow$ Imeening "CONTINUED"). of the eymbol $\nabla$ (meaning "END"). whichever applies.

Mepa, plateo. charo, etc., moy be filmed at different reduction ratios. These too lerge to be entrely inciuded in ons axposure are filmed beginning in the upper inft hand corner, iaft to right and top to bottom, as mamy framee ate required. The following diegrems iliustrate the mothod:
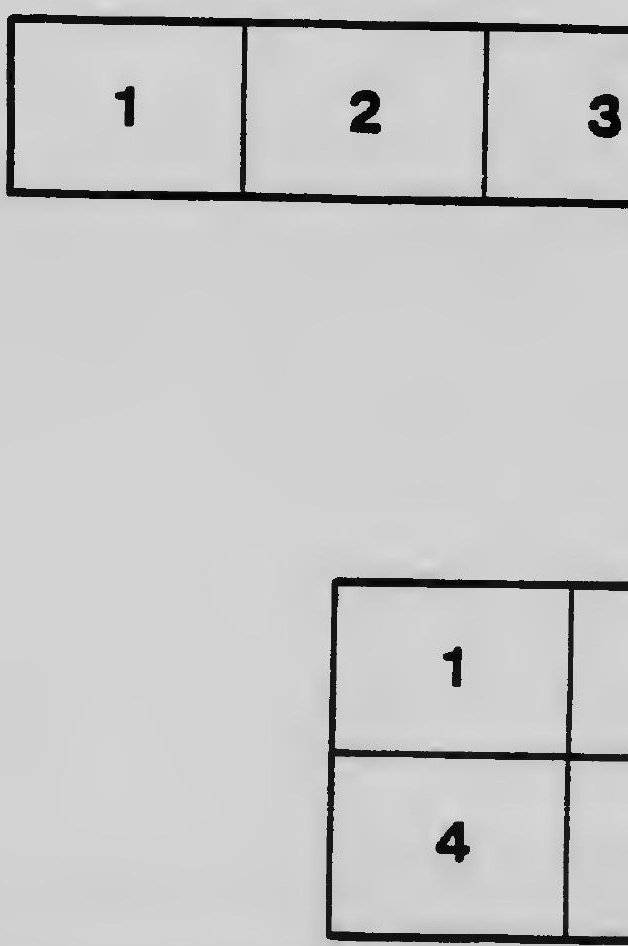
thanks

altry

Ublltity

10

filmed

on

mpres-

All

on the

en-

inted

ON-

"1).

be

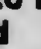

80

the

L'exemplaira films fut reproduit grtee ito ofnśrosltes do:

\section{Bibliothlque \\ Agriculture Canada}

Les images euiventes one the reprodultes ovec is plus grend soin, compte tenu do la condition ot do la notrete de l'exemplaire filmó. ot en conformite eves les conditions du controt de Nimage.

Les exemplaires originaux dont la couverture en papier ast Imprimbe sont filmbe en commeneant par le premior plet ot en terminant solt par la demihro page qul comporte une emprolnte d'Tmprasion ou d'Illustration, soir par lo second plat, selon le cas. Tous les autres exemplalres origlnaux sont filmb en commencent par la premldre pege qul comporte une empreinte d'impreasion ou dillustration ot en terminant par la dernitre pege qul comporte une tolle emprointa.

Un des symboles sutvamta apparaitre sur b demitro image de cheque microfiche. selon is can: lo symbolo - algnifin "A SUIVRE". is srmbole $\nabla$ signifie "FIN".

Lea cartes, plenches, bbleaux, ote. pauvent itre filmes des toux de rduction differents.

Lorsque b decumemt eet trop grand pour stre reproduit on un seul ellehd, II cat filmo d partir do l'angle supiriour gaucho, do gavehe d drole. ot de have on bae. en pronant in nombre d'Tmages nicesalre. Le diagramme sulvants Illustront is mithode.
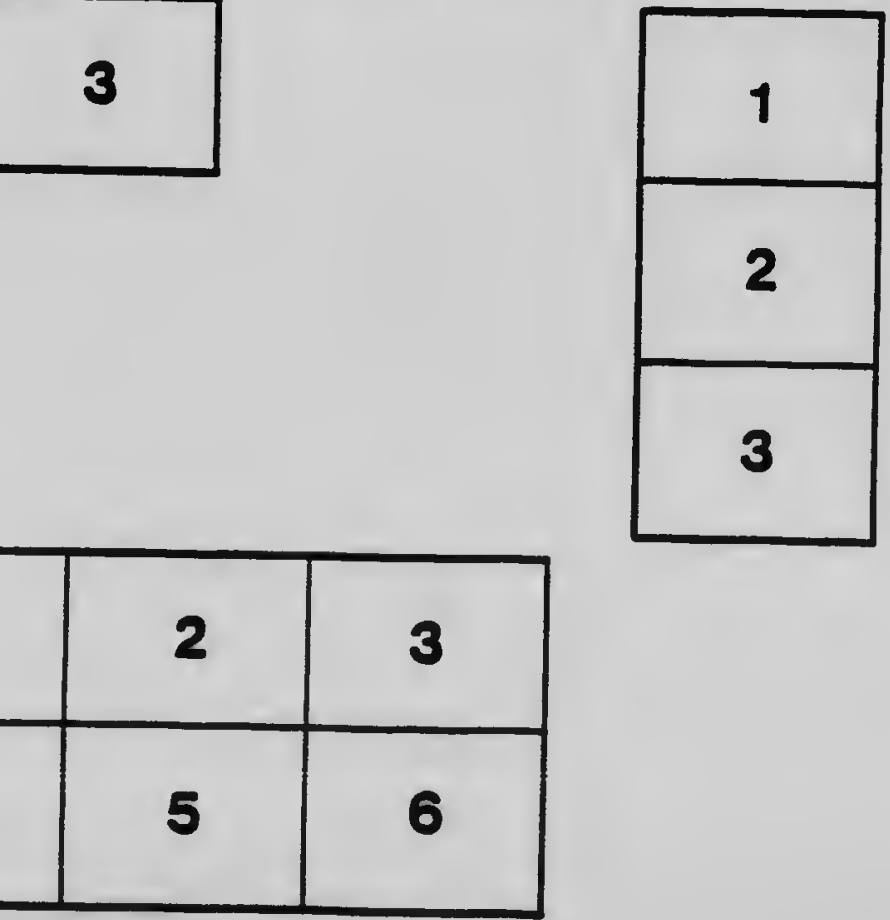


\section{Manocory nESOUTiON TEST CHART}

(ANSI and ISO TEST CHART No. 2)
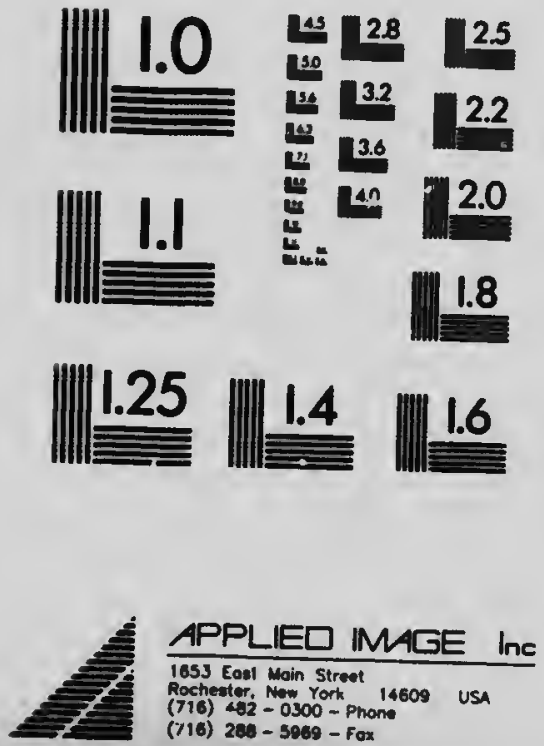
RECEIVED

Alk 3 C 19]"

LANIFRRETE L'AGRICULTURE DE LA PROVINCE DE QUEBE maverelinouticultire

Section Entomologique

\section{BULLETIN No 41}

\section{Traitement des Pommes de Terre et du Blé de Semence}

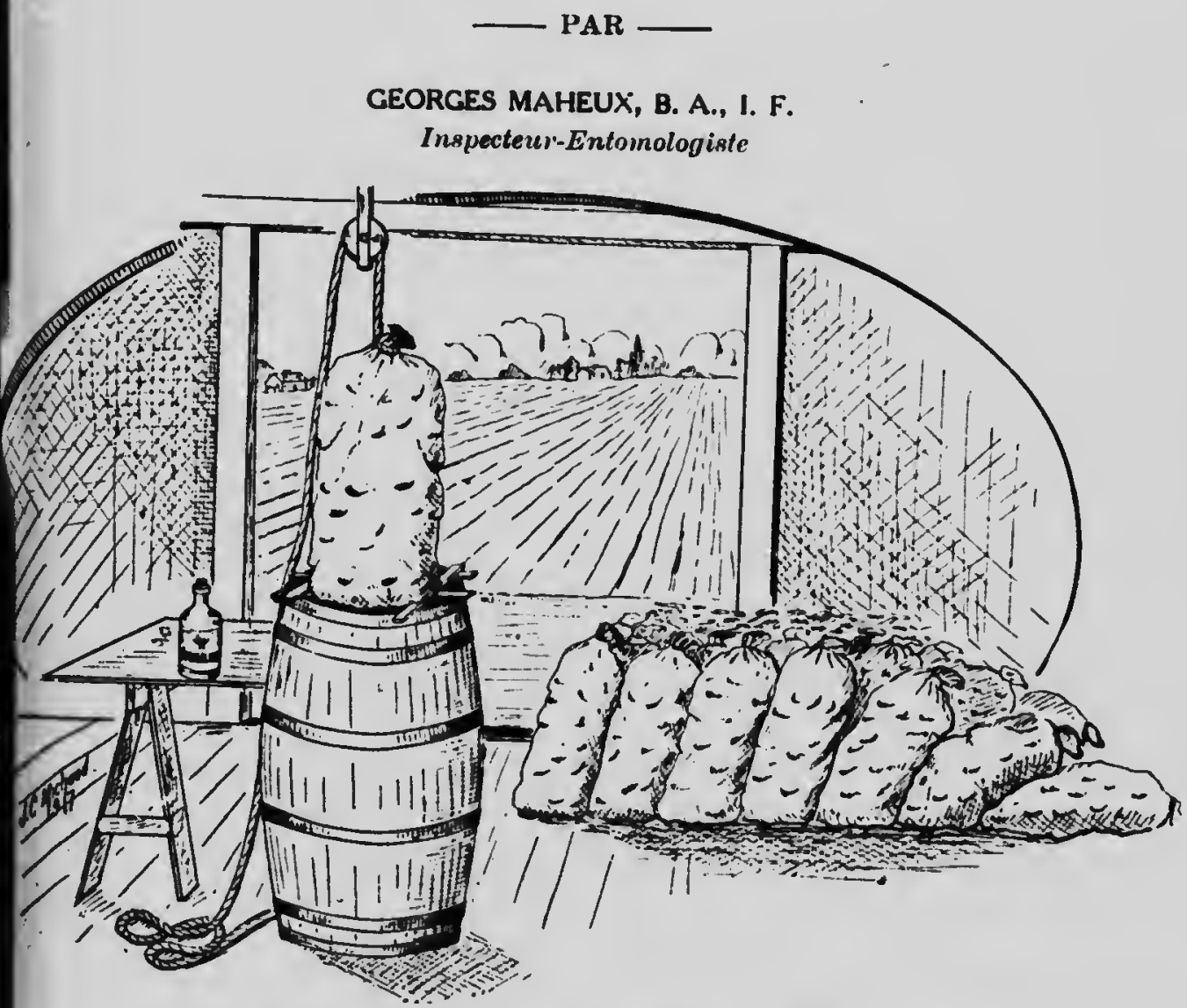

Solution de formalinc dans le baril ; au-dessus, un sac de pommes de terre, mis á égontter sur planches croisées, après le bain.

Poblié par ordre de l'Hon. Jos.-Ed. Caron, Ministre de I'.lgriculture de la proviuce de Québec. 


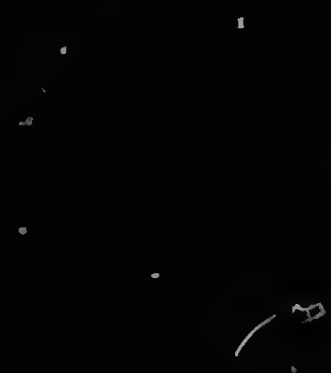

อ

4

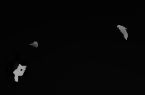

$+$

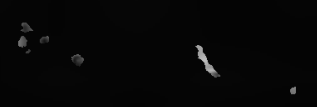

$y=$

$+$ 


\title{
Traitement des Pommes de Terre et du Blé de Semence
}

\author{
I-POMMES DE TERRE
}

\section{But du traitement.}

Grâce aux facilités de transport qui ont multiplié les échanges commerciaux, les eunemis de la pomme de terre ont considérablement augmenté en ces dernières années. Actuellement, nous avons à envisager un grave problènic: combattre énergiquement ces parasites et debarrasser à tout prix nos champs des maladies qui tendent sans cesse a diminuer le rendement de nos récoltes. Ce travail d'enraiement est d'autant plus facile que nous avons à notre disposition des traitements absolument efficaces contre la plupart de ces maladies. Tout cultivateur veut une bonne récolte; il la désire abondante et saine; or, en plusieurs districts de notre pays, cet espoir n'est que chimère si l'horticulteur ne se décide sans retard, à adopter et mettre en pratique le traitement fort simple, mais cxcellent, que nous préconisons plus loin. Ce dernier permet de tuer le mal alors qu'il n'est qu'à l'état de germe asscz inoffensif et assure à celui qui l'emploie des résultats intéressants.

\section{Les maladies.}

1. Gale poudreuse: la plus dangereuse de toutes les maladies que nous trouvons présentement dans le Québec. Les Etats-Unis considèrent cette gale comme tellement grave qu'ils ont pris contre elle des mesures radicales: ainsi, une loi spéciale défend l'innportation de pommes de terre des contrées où cette maladic exerce ses ravagcs; notre province est de ce nombre.

2. Gale commune (maladie des tcrrains gras): très fréquente; il arrive mêmc parfois qu'il soit impossible, en certains endroits, de trouver des tubercules de semence qui n'en soient pas affectés.

3. Petite patate ou coup dc chaleur: accompagne d'ordinaire la gale commune et se rencontre aussi souvent.

4. Pourriture de la cave (sèche et humidc): maladies qui se développent sur les patates pendant l'hiver, clans les caves; affectent souvent la totalité de la provision, rendant les victimes impropres à la plantation.

5. Jambe noire: très répandue, dans les provinces maritimes spécialement, où elle a causé la perte de $90 \%$ de la récolte de 1915 , soit une déperdition de
$\$ 700,000$.

6. Gale noire: sa présence n'a pas encore été signalée ici, bien qu'elle puisse se trouver en quelques cndroits isolés. Les lois provinciale et féclérale class'nt cette maladie parmi les fléaux destructeurs et la décrètent "hors la loi": c'est-àdire, que tout propriétaire d'une récolte atteinte de ce mal ne peut s'en départir en aucune nadnière sans être muni d'un permis émis par l'entomologiste provincial, l'autorisant à cc faire. Elle oblige, en plus, quiconque découvre la gale noire dans son potager à en avertir imniédiatement le ministre de l'agriculture. Toute contravention à cette loi est punissable d'amende $(\$ 100)$. 
7. Mildiou (maladie de la pomme de terre): très répandue partout où l'on cultive ce légume; la partie verte est d'abord attaquée et transmet ensuite l'infection aux tubercules qui deviennent impropres a la consommation; se propage par l'emploi de scmences infec',ées.

8. Pourriture de la tige: se présente, dans la patate, sous forme d'anneau décoloré ou brun-noirátre. Si on sème un tubercule dont l'intérieur est entièrement atteint, il pourrira avant de ger sont impropres aux fins de semence.

9. Mosaiqque: caractérisée sur la plante par des feuilles peu développées recoquillées et desséchées; se transmet par les semences.

Telles sont les maladies de la pomme de terre qui sont transmises par l'emploi de semences affectées; il est facile de reconnaltre les traces des unes ou des autres sur les tubercules au moment de la plantation. Nous avons dit, des le début, que tous les intéressés ont à leur disposition un excellent préventif permettant de restreindre,sinon arrêter complettement, les progrès de ces parasites; ce préventif, c'est le traitement à la formaline qui devrait être employé partout quelle que soit la qualité des semences employées.

\section{Bain de formaline.}

Préparation le matériel essentiel à la préparation du bain de formaline comprend un tonneau, de la formaline et de l'eau. On met dans le tonneau 30 gallons d'eau, puis on y verse une livre ou une chopine de formaline. Si le baril employé n'a pas une contenance de $\mathbf{5 0}$ gallons, il est préférable de ne prendre que la moitié de la formule, soit $\mathbf{1 5}$ gallons d'eau et une demi-livre ou 1 demiard de formaline; autrement, la quantité de pommes de terre qu'on y introduirait ferait renverser la solution. S'il faut traiter rapidement un grand nombre de tubercules, il vaut mieux préparer à l'avance trois, quatre ou cinq barils, selon le cas, de manière a ce que le travail d'ensemencement ne.soit pas retardé.

Exécution: mettez les patates à semer encore rondes dans des sacs bien propres afin de ne pas troubler la solution et de permettre au liquide de pénétrer rapidement jusqu'aux pommes de terre. A l'aide d'une poulie et d'une corde, plongez le sac rempli dans le baril: laissez baigner les semences pendant deux heures entières soulevez ensuite et mettez a égoutter au-dessus du baril en ayant soin d'àppuyer le sac sur de petites planches croisées, tel que le fait voir la gravure frontispice.

Cette opération terminée,versez les tubercules sur le gazon ou sur des planches. L'important est que tout ce dont on se scrvira pour la manipulation des semences désinfectées soit rigoureusement propre, agir autrement serait compromettre le succès du traitement en mettant ces semences en contact avec des objets chargés de germes de maladies. Lorsq̣ue les pommes de terre sont séchécs, on peut procéder a la taille des germes ou plantons. Pour ce travail, il est nécessaire que chaque ouvrier ait deux ou trois couteaux a sa disposition; pendant qu'il se sert de l'un d'eux, les autres baignent dans une bouteille ou vase renfer- 
nant une forte solution de formaline et même de formaline pure. S'il arrive que l'on tranche une pomme ..e terre dont la chair laisse voir des traces de nialadies, on met clans la forınaline la lame qui a certainement entralné des gernıes du mal et on prend, en échange, un des couteaux désinfectés.

L'emploi de la formaline ne présente par de danger, puisque ce n'est, pas un poison violent; son seul inconvénient est d'irriter la peau; au gout elle est nettement acre.

\section{Remarques.} possible.

On ne doit employer pour la semence que des tubercules aussi sains que

Quelle que soit la qualité des semences employées, il ne faut jamais négliger de les passer au bain de formaline; elles peuvent fort bien contenir des gernies que nous n'apercevons pas.

Ce traitement ne renferme pas en soi la somme de soins que le cultivateur doit donner aux pommes de terre; non, lorsque les plants seront levés, qu'ils possèderont tiges et feuilles, il ne faudra pas négliger les arrosages a la bouillie bordelaise. (Voir bulletin No 37 pour explications.)

Plus vous prendrez de soins pour protéger vos pommes de terre contre les maladies, plus le rendement sera élevé en nombre et en qualité; conséquemment les revenus que vous retirerc, de cette culture seront plus forts et vous dédommageront amplement de la peine que vous vous serez donnée.

Ne plantez jamais des pommes de terre sur un sol qui a déjà donné une récolte contaminée: ce serait courir au-devant de l'insuccès.

Enfin, ne ... ligez rien pour produire des légumes de première qualité: il y va de votre intérêt et de la bonne renommée de votre province.

(Le ministère de l'agriculture d'Ottawa distribue gratuitement plusieurs bulletins et circulaires traitant des maladies de la pomme de terre).

\section{II-LE BLE}

\section{Maladies.}

Cette précieuse céréale est victime de deux graves malaries: la carie et le eharbon.

\section{Traitement.}

Le traitement de la carie est le même que celui de la pomme de terre: 30 gallons d'eau et une livre de formaline, ou une demi-livre pour 15 gallons; la seule différence réside dans la durée du bain; il n'est, dans le cas de la carie, que de 5 minutes. On fait sécher ensuite et on sème aussitôt.

Le bulletin No 26, intitule "Le ble" et écrit par M. F.-N. Savoie indique d'une manière complète le traitement du charbon: nous y renvoyong nos lecteurs. (Gratis sur demande au ministère de l'agriculture à Québec). 
y

in

3 


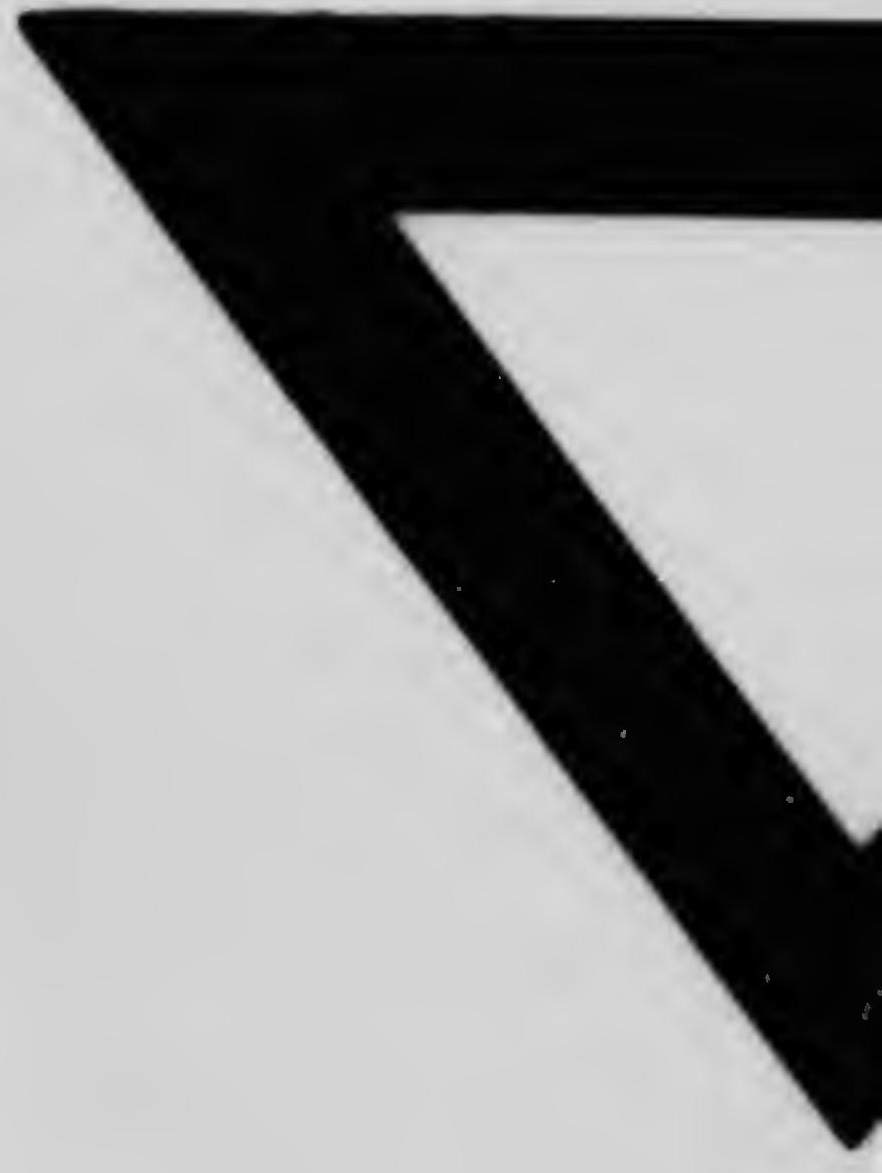




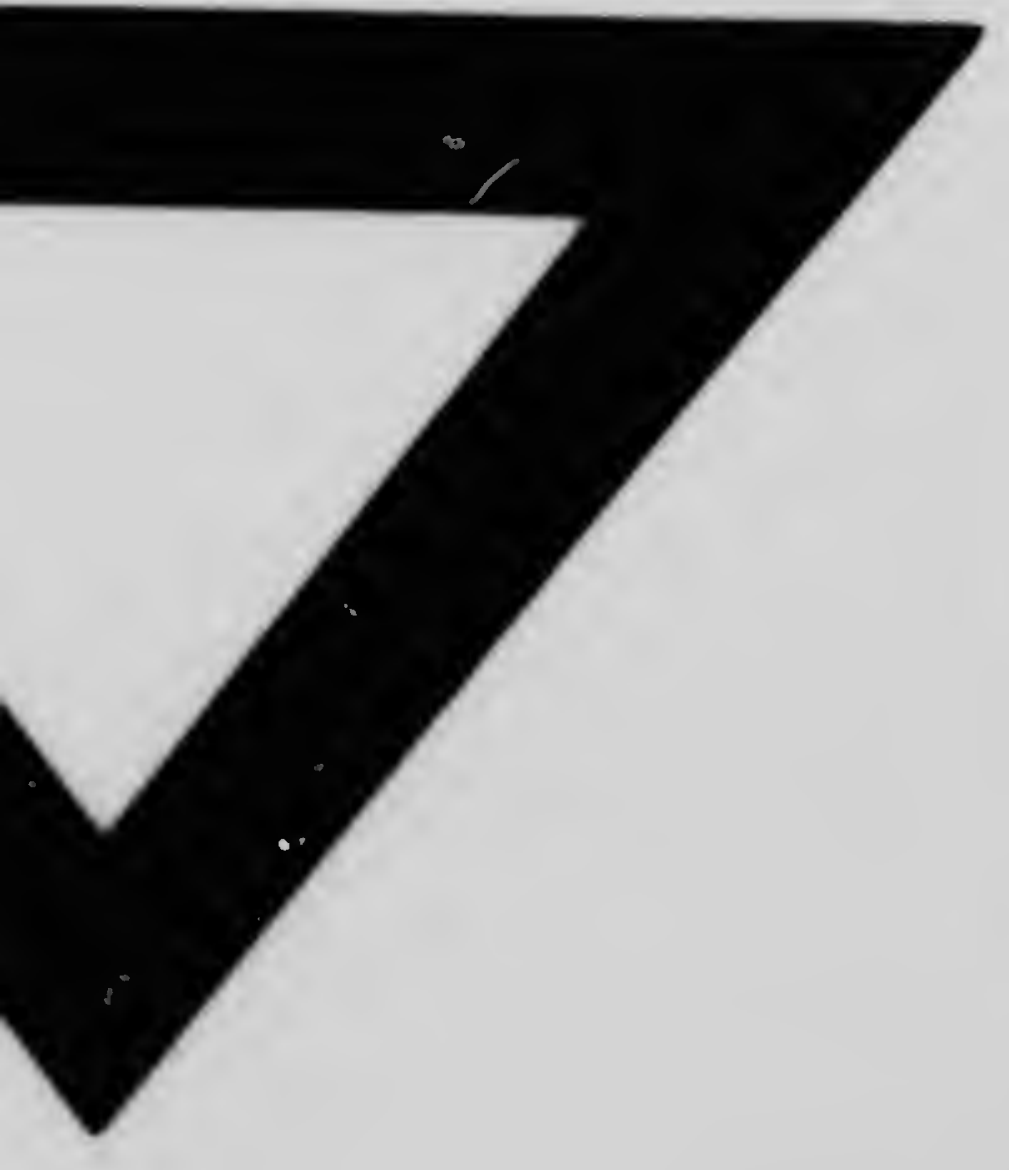

